\title{
EXPONENTIAL MODEL OF TOKEN BUCKET SYSTEM
}

In the presented paper we use the Theory of Markovov Chains for simulation of a simple model of the Token Bucket System (TBS). In the first section we deal with a steady-state analysis of the TBS with Exponential model of On-Off Source of human speech. We develop recurrent formulas for state probabilities of the TBS. In the second section we use real parameters of VoIP and we calculate characteristic of the TBS. In the last section the values of probability of a loosing packet are approximated by more types of regression functions. It is shown that the cubic approximation is the most efficient. Using this function we can directly compute values of probability of a loosing packet.

\section{Steady-state Analysis}

The Token Bucket System is related to VoIP problems. We have formed the steady-state analysis of VoIP under the Token Bucket Control. Our main problems will be to compute the probability characteristics of TBS and to find relation between probability of a loosing packet and the bucket depth. At first we will analyze the work of TBS in general (Fig. 1).

$$
\begin{aligned}
\text { ON: } T_{1} \sim f_{1}(t) & =\alpha e^{-\alpha t} \\
E T_{1}=\frac{1}{\alpha} & =227 \mathrm{~ms} \quad \alpha=0.00441 \mathrm{~ms}^{-1} \\
\text { OFF: } T_{2} \sim f_{2}(t) & =\mu e^{-\mu t} \\
E T_{1}=\frac{1}{\mu} & =596 \mathrm{~ms} \quad \alpha=0.00168 \mathrm{~ms}^{-1}
\end{aligned}
$$

\section{$\lambda$ - token rate}

\section{$\eta$ - packet rate}
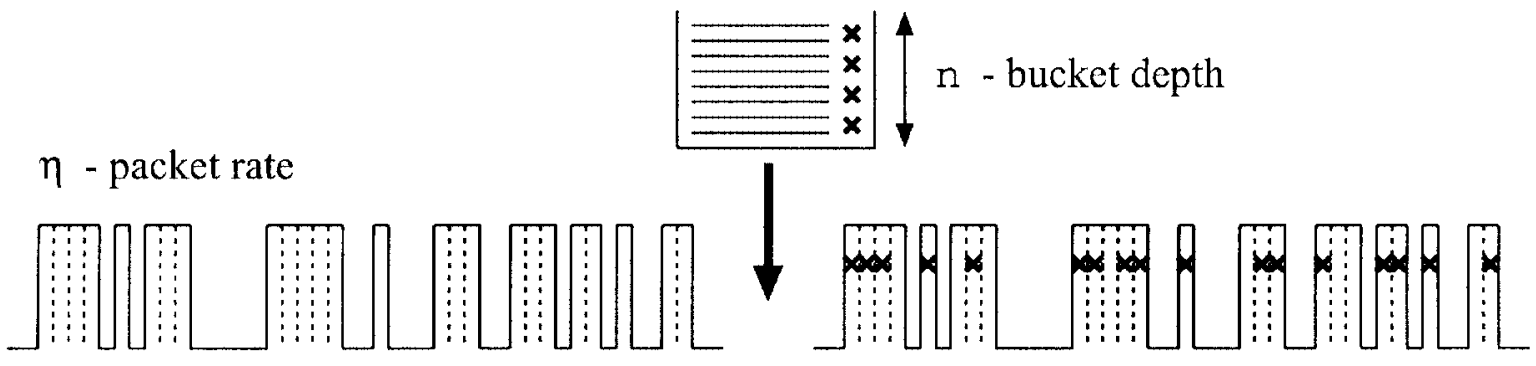

Fig. 1

There is a flow of packets entering the TBS. The Token Bucket System is generating tokens (marks) and then it marks each packet with one of them. Only marked packets will go through the network. For practical reasons we can assume a limited bucket with depth " $n$ ". In case the bucket is empty (there are no tokens), TBS cannot mark any packet, which is lost then. Let $P_{l s t}$ be probabilities of this random phenomenon and we will call it "probability of loosing packet”.

We will deal with simple Exponential model of On-Off Source of human speech. This Source has two states. The state On is period of "speech" and the Off state is period of "silence". Between these states, the source switches randomly. Talk-spurt duration is modelled by variant $T_{1}$ and pause duration is modelled by variant $T_{2}$. The values of distribution parameters were gained from [1].
When the Source is in On state it starts generating a flow of packets, which represents human speech. The packet rate in usual VoIP systems (using G.729A) is $50 \mathrm{p} / \mathrm{s}$. We will assume that the flow of packets is modelled by Poisson process $N_{P}(t)$ with rate $\eta=0.05 \mathrm{p} / \mathrm{ms}$.

In general there can be any token rate. Usually it is the same as an average number of packets entering the TBS. Let $P_{O N}$ and $P_{\text {OFF }}$ be probabilities that the Source is in state On or Off. The flow of tokens will be modelled by Poisson process $N_{T}(t)$ with rate $\lambda=\eta \cdot P_{\text {ON }}+0 . P_{\text {OFF }}$.

We will construct a transition diagram of Markov model of On-Off Source (Fig. 2):

\footnotetext{
* Juraj Smieško

Department of InfoCom Networks, Faculty of Management Science and Informatics, University of Žilina
} 


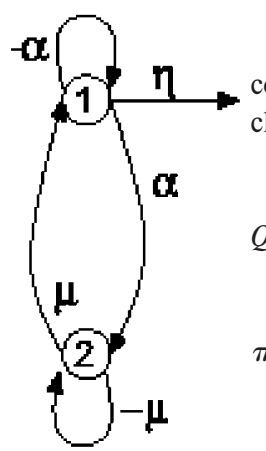

Let $Q_{0}$ be a rate matrix for this chain. We compute state probabilities $\pi$ of a steady-state chain by balance equations:

$Q_{0}=\left(\begin{array}{rr}-\alpha & \alpha \\ \mu & -\mu\end{array}\right) \quad \pi=\left(\pi_{1}, \pi_{2}\right)$,

$\pi \cdot Q_{0}=0 \Rightarrow \pi=\left(\frac{\mu}{\alpha+\mu}, \frac{\alpha}{\alpha+\mu}\right)$

Fig. 2

We compute probabilities and token rate $\pi=(0.27586,0.72414)$ for real parameters of VoIP:

$\mu=0.05 \mathrm{p} / \mathrm{ms} . P_{O N}=0.05 \mathrm{p} / \mathrm{ms} . \pi_{1}=0.01379 \mathrm{p} / \mathrm{ms}$

In the Token Bucket System we have three elementary random phenomena, entering the packet, generating token and switching between On-Off states in Source. We have assumed that each of them is Poisson process, and because of that we can model this TBS by Markov chain.

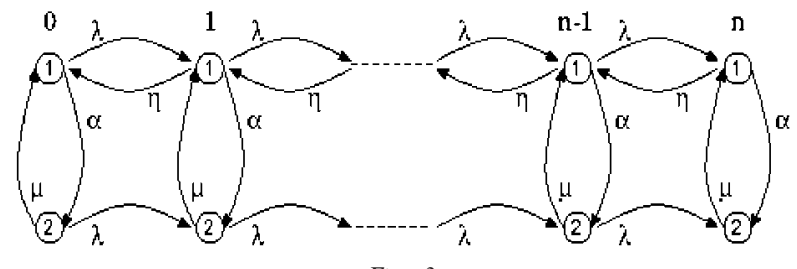

Fig. 3

Probability of an increasing number of tokens in the bucket about one during "short" time $\Delta t$ is

$$
Q^{\prime}=\left(\begin{array}{ccccccccc}
-\mu & \alpha & \lambda & 0 & \ldots & 0 & 0 & 0 & 0 \\
\mu & -(\alpha+\eta) & 0 & \lambda & \ldots & 0 & 0 & 0 & 0 \\
0 & 0 & -(\lambda+\mu) & \alpha & \ldots & 0 & 0 & 0 & 0 \\
0 & \eta & \mu & -(\lambda+\alpha+\eta) & \ldots & 0 & 0 & 0 & 0 \\
\vdots & \vdots & \vdots & \vdots & \ddots & \vdots & \vdots & \vdots & \vdots \\
0 & 0 & 0 & 0 & \ldots & -(\lambda+\mu) & \alpha & \lambda & 0 \\
0 & 0 & 0 & 0 & \ldots & \mu & -(\lambda+\alpha+\eta) & 0 & \lambda \\
0 & 0 & 0 & 0 & \ldots & 0 & 0 & -(\lambda+\mu) & \alpha \\
0 & 0 & 0 & 0 & \ldots & 0 & \eta & \mu & -(\lambda+\alpha)
\end{array}\right)
$$

We modified the rate matrix $Q^{\prime}$ to the equivalent triangular matrix and we used a normalization condition for state probabilities $\sum_{k=0}^{n}\left(X_{k}+Y_{k}\right)=1$

$$
\left(\begin{array}{ccccccccc}
-\mu & \alpha & \lambda & 0 & \ldots & 0 & 0 & 0 & 0 \\
0 & -\eta & \lambda & \lambda & \ldots & 0 & 0 & 0 & 0 \\
0 & 0 & -(\lambda+\mu) & \alpha & \ldots & 0 & 0 & 0 & 0 \\
0 & 0 & 0 & -\eta & \ldots & 0 & 0 & 0 & 0 \\
\vdots & \vdots & \vdots & \vdots & \ddots & \vdots & \vdots & \vdots & \vdots \\
0 & 0 & 0 & 0 & \ldots & -(\lambda+\mu) & \alpha & \lambda & 0 \\
0 & 0 & 0 & 0 & \ldots & 0 & -\eta & \lambda & \lambda \\
0 & 0 & 0 & 0 & \ldots & 0 & 0 & -(\lambda+\mu) & \alpha \\
1 & 1 & 1 & 1 & \ldots & 1 & 1 & 1 & 1
\end{array}\right) \cdot\left(\begin{array}{c}
Y_{n} \\
X_{n} \\
Y_{n-1} \\
X_{n-1} \\
\vdots \\
Y_{1} \\
X_{1} \\
Y_{0} \\
X_{0}
\end{array}\right)=\left(\begin{array}{c}
0 \\
0 \\
0 \\
0 \\
\vdots \\
0 \\
0 \\
0 \\
1
\end{array}\right)
$$


We gained the recurrent formulas for state probabilities:

$Y_{0}=\frac{\alpha}{\lambda+\mu} X_{0}, \quad X_{k}=\frac{\lambda}{\eta}\left[Y_{k-1}+X_{k-1}\right]$ for $k=1, \ldots, n$

$Y_{k}=\frac{\alpha}{\lambda+\mu} X_{k}+\frac{\lambda}{\lambda+\mu} Y_{k-1}$ for $k=1, \ldots, n$,

$Y_{n}=\frac{\alpha}{\mu} X_{n}+\frac{\lambda}{\mu} Y_{n-1}$

We can reduce formulas for $Y_{k}$ and $Y_{n}$ to:

$X_{k}=\frac{\lambda}{\lambda+\mu}\left[\frac{\alpha+\eta}{\eta} Y_{k-1}+\frac{\alpha}{\eta} X_{k-1}\right]$ for $k=1, \ldots n$,

$Y_{n}=\frac{\lambda}{\mu}\left[\frac{\alpha+\eta}{\eta} Y_{n-1}+\frac{\alpha}{\eta} X_{n-1}\right]$

\section{TBS with real parameters of VoIP}

To deduce explicit formula for probability of loosing packet $P_{l s t}=P_{l s t}(n)=X_{0}$ is difficult and impracticle, but there is no problem to compute the values of $P_{l s t}$ for real parameters of our TBS with Exponential On-Off Source:

$$
\begin{aligned}
& \alpha=0.00441 \mathrm{~m} . \mathrm{s}^{-1} \quad \mu=0.00168 \mathrm{~m} . \mathrm{s}^{-1} \\
& \lambda=0.01379 \mathrm{p} / \mathrm{ms} \quad \eta=0.05 \mathrm{p} / \mathrm{ms}
\end{aligned}
$$

Than recurrent formulas for state probabilities are formed as:

$$
\begin{aligned}
& Y_{0}=0.28507 \cdot X_{0} \quad X_{k}=0.27580 \cdot\left[Y_{k-1}+X_{k-1}\right] \\
& \text { for } k=1, \ldots, n, \\
& Y_{k}=0.97002 \cdot Y_{k-1}+0.07862 \cdot X_{k-1} \quad \text { for } k=1, \ldots, n, \\
& Y_{n}=8.93231 \cdot Y_{n-1}+0.72397 \cdot X_{n-1}
\end{aligned}
$$

For example, the reader can see the difference between $n=4$ and $n=5$ :

\begin{tabular}{|c|c|c|c|}
\hline$n$ & $X_{k}$ & $Y_{k}$ & $P_{k}$ \\
\hline 0 & 0.14880 & 0.04242 & 0.19122 \\
\hline 1 & 0.05274 & 0.05285 & 0.10559 \\
\hline 2 & 0.02912 & 0.05541 & 0.08453 \\
\hline 3 & 0.02331 & 0.05604 & 0.07935 \\
\hline 4 & 0.02188 & 0.51742 & 0.53931 \\
\hline
\end{tabular}

\begin{tabular}{|c|c|c|c|}
\hline$n$ & $X_{k}$ & $Y_{k}$ & $P_{k}$ \\
\hline 0 & 0.13803 & 0.03935 & 0.17738 \\
\hline 1 & 0.04892 & 0.04902 & 0.09794 \\
\hline 2 & 0.02701 & 0.05140 & 0.07841 \\
\hline 3 & 0.02136 & 0.05198 & 0.07361 \\
\hline 4 & 0.02030 & 0.05212 & 0.07242 \\
\hline 5 & 0.01997 & 0.48027 & 0.50024 \\
\hline
\end{tabular}

For real use its enough to have the bucket depth $n=1, \ldots, 10$. Now we will increase the bucket depth $n$ and calculate characteristics of models:

$P_{I s t}(n)$ - probability of loosing packet or probability of empty bucket in time of arriving packet

$\lambda . P_{l s t}(n)$ - average number of lost packets

$P_{n}$ - probability of full bucket (bucket will refuse tokens)

$E K$ - average bucket depth $\aleph=\frac{E K}{n}-$ token bucket usage

\begin{tabular}{|c|c|c|c|c|c|}
\hline$n$ & $P_{l s t}(n)$ & $\lambda \cdot P_{l s t}(n)$ & $P_{n}$ & $E K$ & $\aleph$ \\
\hline 1 & 0.20367 & $2.809 \mathrm{p} / \mathrm{s}$ & 0.73826 & 0.73826 & $73.8 \%$ \\
\hline 2 & 0.17796 & $2.454 \mathrm{p} / \mathrm{s}$ & 0.64503 & 1.41634 & $70.8 \%$ \\
\hline 3 & 0.16163 & $2.229 \mathrm{p} / \mathrm{s}$ & 0.58580 & 2.05572 & $68.5 \%$ \\
\hline 4 & 0.14880 & $2.052 \mathrm{p} / \mathrm{s}$ & 0.53931 & 2.66994 & $66.7 \%$ \\
\hline 5 & 0.13803 & $1.903 \mathrm{p} / \mathrm{s}$ & 0.50024 & 3.26649 & $65.3 \%$ \\
\hline 6 & 0.12874 & $1.775 \mathrm{p} / \mathrm{s}$ & 0.46658 & 3.84967 & $64.2 \%$ \\
\hline 7 & 0.12064 & $1.664 \mathrm{p} / \mathrm{s}$ & 0.43719 & 4.42225 & $63.2 \%$ \\
\hline 8 & 0.11349 & $1.565 \mathrm{p} / \mathrm{s}$ & 0.41129 & 4.98619 & $62.3 \%$ \\
\hline 9 & 0.10715 & $1.478 \mathrm{p} / \mathrm{s}$ & 0.38828 & 5.54295 & $61.6 \%$ \\
\hline 10 & 0.10148 & $1.399 \mathrm{p} / \mathrm{s}$ & 0.36771 & 6.09369 & $60.9 \%$ \\
\hline
\end{tabular}

\section{Relation between Probability of loosing packet and the Token Bucket depth}

The most interesting characteristic is probability of loosing packet $P_{l s t}(n)$. Its values will be approximated by regression functions $Y_{I}(n)$ :

$$
\begin{array}{ll}
Y_{1}(n)=a n+b & Y_{2}(n)=a n^{2}+b n+c \\
Y_{3}(n)=a n^{3}+b n^{2}+c n+d & Y_{e}(n)=a e^{b n}
\end{array}
$$

by the least squares method. We will measure the quality of approximation by square of sum residuals:

$$
\breve{S}_{I}=\sqrt{\sum_{n=0}^{10}\left[P_{l s t}(n)-Y_{I}(n)\right]^{2}} \quad I=1,2,3, e
$$


Approximation by the linear function: $Y_{1}(n)=-0.01060 . n+0.19849$ with $\quad \mathfrak{S}_{1}=0.02307$

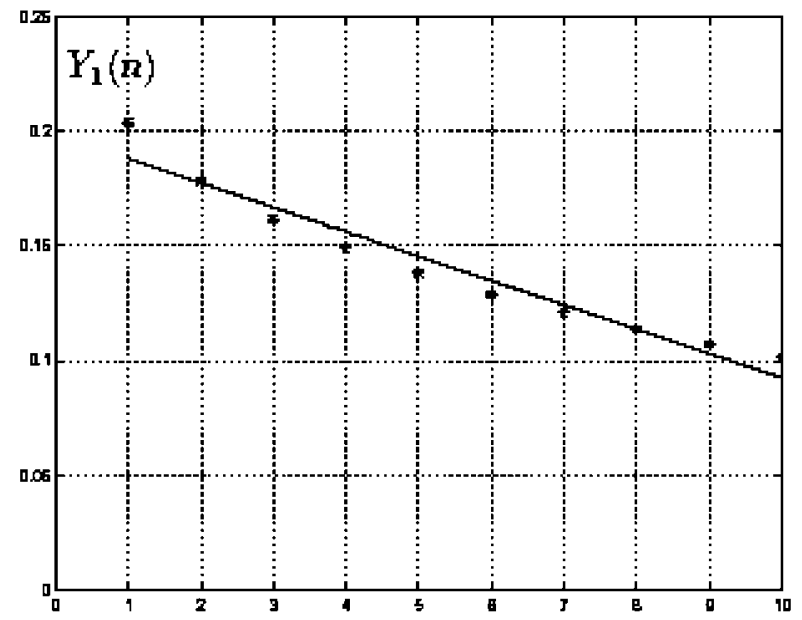

\begin{tabular}{|c|c|c|}
\hline$n$ & $Y_{l}(n)$ & $P_{l s t}(n)-Y_{I}(n)$ \\
\hline 1 & 0.18788 & 0.01579 \\
\hline 2 & 0.17728 & 0.00068 \\
\hline 3 & 0.16667 & 0.00505 \\
\hline 4 & 0.15607 & 0.00726 \\
\hline 5 & 0.14546 & 0.00743 \\
\hline 6 & 0.13486 & 0.00611 \\
\hline 7 & 0.12425 & 0.00361 \\
\hline 8 & 0.11365 & 0.00015 \\
\hline 9 & 0.10304 & 0.00411 \\
\hline 10 & 0.09244 & 0.00904 \\
\hline
\end{tabular}

Quadratic regression: $Y_{2}(n)=0.00095 \cdot n^{2}-0.02105 \cdot n+0.21937$

with $\quad \mathfrak{C}_{2}=0.00753$

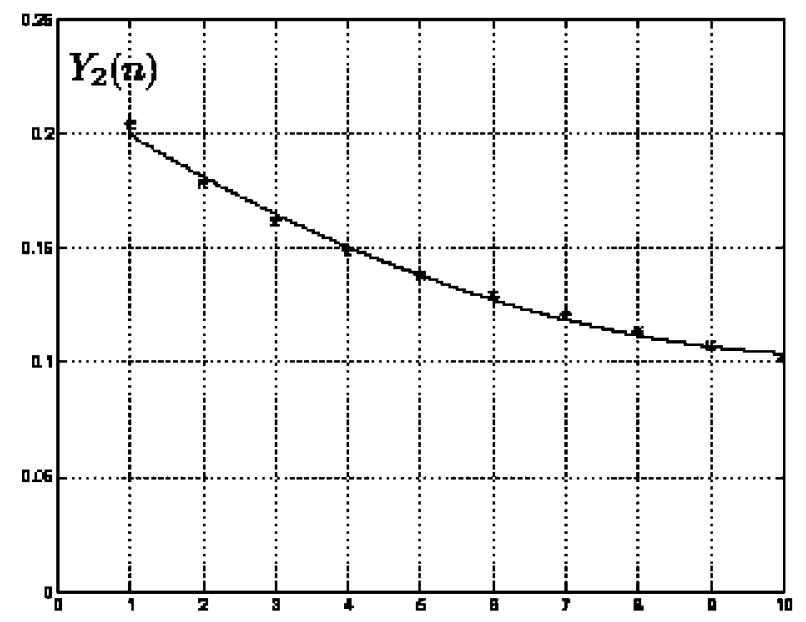

\begin{tabular}{|c|c|c|}
\hline$n$ & $Y_{2}(n)$ & $P_{l s t}(n)-Y_{2}(n)$ \\
1 & 0.19927 & 0.00440 \\
\hline 2 & 0.18108 & -0.00311 \\
\hline 3 & 0.16477 & -0.00315 \\
\hline 4 & 0.15037 & -0.00157 \\
\hline 5 & 0.13787 & 0.00016 \\
\hline 6 & 0.12726 & 0.00148 \\
\hline 7 & 0.11856 & 0.00208 \\
\hline 8 & 0.11175 & 0.00175 \\
\hline 9 & 0.10684 & 0.00031 \\
\hline 10 & 0.10383 & -0.00235 \\
\hline
\end{tabular}

Cubic regression: $Y_{3}(n)=-0.00012 \cdot n^{3}+0.00297 \cdot n^{2}-0.03039 \cdot n+0.22990$ with $\mathscr{S}_{3}=0.00318$

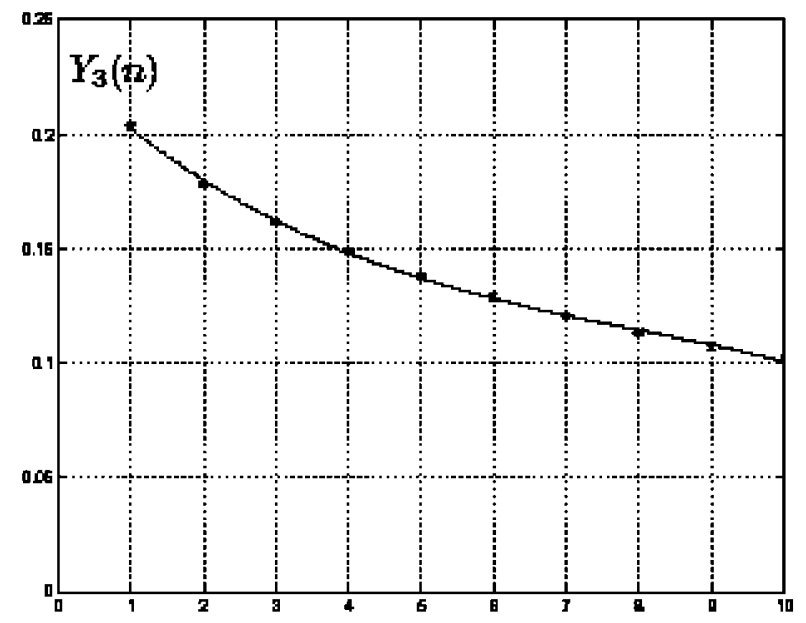

\begin{tabular}{|c|c|c|}
\hline$n$ & $Y_{3}(n)$ & $P_{\text {lst }}(n)-Y_{3}(n)$ \\
\hline 1 & 0.20237 & 0.00131 \\
\hline 2 & 0.18004 & -0.00208 \\
\hline 3 & 0.16220 & -0.00057 \\
\hline 4 & 0.14809 & 0.00071 \\
\hline 5 & 0.13697 & 0.00104 \\
\hline 6. & 0.12815 & 0.00060 \\
\hline 7. & 0.12084 & -0.00020 \\
\hline 8 & 0.11432. & -0.00083 \\
\hline 9 & 0.10787 & -0.00072 \\
\hline 10 & 0.10073 & 0.00074 \\
\hline
\end{tabular}




\section{kam Nikcale}

Approximation by the exponential function: $Y_{e}(n)=0.20615 \cdot e^{-0.07447}$

with $\quad \mathfrak{C}_{e}=0.01501$

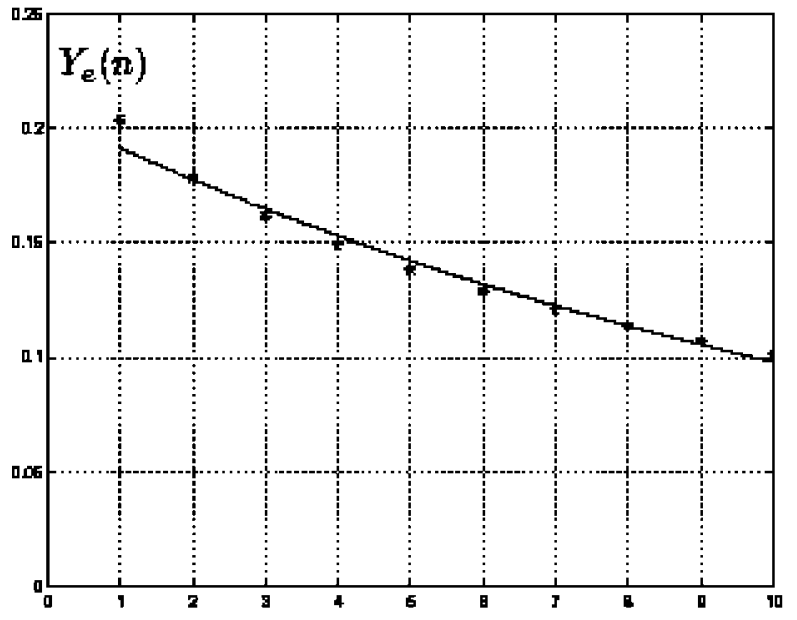

\begin{tabular}{|cc|c|}
\hline$n$ & $Y_{e}(n)$ & $P_{l s t}(n)-Y_{e}(n)$ \\
\hline 1 & 0.19135 & 0.01232 \\
\hline 2 & 0.17762 & 0.00034 \\
\hline 3 & 0.16487 & -0.00325 \\
\hline 4 & 0.15304 & -0.00424 \\
\hline 5 & 0.14206 & -0.00403 \\
\hline 6 & 0.13186 & -0.00312 \\
\hline 7 & 0.12240 & -0.00176 \\
\hline 8 & 0.11361 & 0.00012 \\
\hline 9 & 0.10546 & 0.00169 \\
\hline 10 & 0.09789 & 0.00359 \\
\hline
\end{tabular}

We can see the cubic regression function is a very "good" approximation with maximum error $2,1 \cdot 10^{-3}$. If we are satisfied with this precision, we can exchange $P_{l s t}(n)$ for $Y_{3}(n)$ :

$P_{l s t}(n) \doteq-0.00012 \cdot n^{3}+0.00297 \cdot n^{2}-0.03039 \cdot n+0.22990$

Because of its practical use the exponential approximation is "better" (with maximum error $1,2 \cdot 10^{-2}$ ).

\section{References}

[1] ITV-T Recommendation P.59: Articial Conversational Speech, 1993

[2] PEŠKO \& SMIEŠKO: Stochastic Models of Operation Research (Stochastické Modely Operačnej Analýzy), Žilinská univerzita, 1999. 